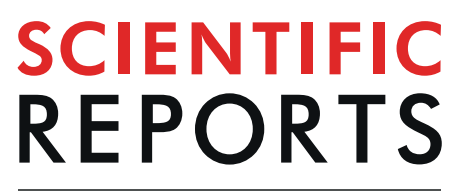

natureresearch

\title{
MBW complexes impinge on anthocyanidin reductase gene regulation for proanthocyanidin biosynthesis in persimmon fruit
}

\author{
Francisco Gil-Muñoz ${ }^{1}$, Jesús A. Sánchez-Navarro ${ }^{2}$, Cristina Besada ${ }^{1}$, Alejandra Salvador ${ }^{1}$, \\ María Luisa Badenes ${ }^{1}$, María del Mar Naval ${ }^{1} \&$ Gabino Ríos $^{1 *}$
}

MBW protein complexes containing MYB, bHLH and WD40 repeat factors are known transcriptional regulators of secondary metabolites production such as proanthocyanidins and anthocyanins, and developmental processes such as trichome formation in many plant species. DkMYB2 and DkMYB4 (MYB-type), DkMYC1 (bHLH-type) and DkWDR1 (WD40-type) factors have been proposed by different authors to take part of persimmon MBW complexes for proanthocyanidin accumulation in immature fruit, leading to its characteristic astringent flavour with important agronomical and ecological effects. We have confirmed the nuclear localization of these proteins and their mutual physical interaction by bimolecular fluorescence complementation analysis. In addition, transient expression of DKMYB2, $D K M Y B 4$ and $D K M Y C 1$ cooperatively increase the expression of a persimmon anthocyanidin reductase gene $(A N R)$, involved in the biosynthesis of cis-flavan-3-ols, the structural units of proanthocyanidin compounds. Collectively, these data support the presence of MBW complexes in persimmon fruit and suggest their coordinated participation in $A N R$ regulation for proanthocyanidin production.

Proanthocyanidins (PAs), or condensed tannins, are flavonoid polymers that accumulate in fruits, leaves, seeds and other tissues of many plants, providing protection against pathogens and herbivores. PAs also contribute to fruit flavour and colour and are considered beneficial for human health in virtue of their antioxidant properties, among other salutary attributes ${ }^{1}$. In persimmon (Diospyros kaki), the microstructure and accumulation of soluble PAs of the so called tannin cells has been found related to fruit astringent taste ${ }^{2,3}$, potentially affecting fruit palatability for frugivorous animals ${ }^{4}$. Interestingly, soluble tannins are reduced throughout persimmon fruit development and maturation ${ }^{3,5}$, providing a way to channel the action of frugivores when seeds are fully viable and ready for dispersal. In addition to a poorly known mechanism involving the transcriptional repression of PA biosynthetic enzymes ${ }^{6}$, soluble PA content is reduced during fruit ripening by the production of acetaldehyde by seeds, leading to PA insolubilization and the subsequent astringency loss ${ }^{7,8}$. Although the content of soluble tannins becomes undetectable from a sensory point of view at overripening stages, the concomitant loss of fruit firmness importantly limits fruit postharvest life and therefore the commercialization opportunities. To overcome this limitation, the fruit is harvested before overripening and subjected to deastringency postharvest treatments to remove astringency while maintaining high firmness ${ }^{9}$. Most of deastringency methods in persimmon are based on maintaining the fruit under anaerobic conditions or exposing them to products that induce anaerobic respiration. Under these conditions, soluble tannins are polymerized by acetaldehyde accumulated in the flesh ${ }^{10,11}$. Furthermore, natural non-astringent mutants exist into persimmon germplasm collections. Several of these non-astringent cultivars are hypothesized to carry a recessive mutation in a single gene known as $A S T^{12,13}$, but the molecular function and identity of this gene remain unknown. Postharvest treatments of astringent varieties improve the postharvest life and the organoleptic quality of treated fruit, however these treatments represent an important production cost and are often a challenge for new varieties or stressed orchards ${ }^{14}$. Thus, studying PAs biosynthesis and metabolism in persimmon fruit may help to better understand the different mechanisms

${ }^{1}$ Instituto Valenciano de Investigaciones Agrarias (IVIA), E-46113 Moncada, Valencia, Spain. ${ }^{2}$ Instituto de Biología Molecular y Celular de Plantas, Universidad Politécnica de Valencia-CSIC, E-46022, Valencia, Spain. *email: rios_ gab@gva.es 
employed by plants to drive frugivore-dependent dispersal of seeds, and to improve deastringency treatments and crop management with the aim to reduce costs and increase the sustainability of persimmon production.

The pathway of PAs biosynthesis has been genetically dissected by the analysis of different seed mutants in Arabidopsis thaliana, barley and maize among other species ${ }^{15}$. PAs are formed by condensation of trans- and cis-flavan-3-ols units, synthesized respectively by stereospecific leucoanthocyanidin reductase (LAR) and anthocyanidin reductase (ANR) enzymes ${ }^{16,17}$. These PA biosynthetic activities and genes are essentially conserved in persimmon, with some regulatory particularities ${ }^{18}$. In persimmon fruit, $A N R$ gene is much more expressed than its counterpart $L A R$, consistent with the higher content of cis-flavan-3-ols stereoisomers in PA composition ${ }^{19}$. In addition, $A N R$ is strongly repressed in advanced steps of fruit maturation, concomitantly with tannin decrease, which points to a role of $A N R$ as a major integrative target of PA regulatory pathways in persimmon.

In Arabidopsis, regulation of the ANR orthologous gene BANYULS (BAN) and PA accumulation in seed coat requires the concerted action of TRANSPARENT TESTA2 (TT2), TRANSPARENT TESTA8 (TT8) and TRANSPARENT TESTA GLABRA1 (TTG1), encoding respectively a R2R3-MYB transcription factor, a basic helix-loop-helix (bHLH) transcription factor and a WD40-repeat (WDR) protein ${ }^{20}$. These regulatory factors form a ternary complex named MYB-bHLH-WD40 (MBW) that may invoke the participation of alternative MYB and bHLH components for the regulation of particular steps of PA and anthocyanin biosynthetic pathways ${ }^{21,22}$. MBW complexes also contribute to PAs production in edible fruits of crop plants such as grapevine ${ }^{23}$, strawberry ${ }^{24}$, apple $^{25}$ and persimmon ${ }^{26}$.

In persimmon, DkMYB2 and DkMYB4 genes cause an altered pattern of PAs accumulation and expression of biosynthetic enzymes when misexpressed, and increase ANR promoter transcriptional activity in transient reporter assays when combined with a heterologous bHLH factor from Arabidopsis ${ }^{27,28}$. Interestingly, DkMYB2 and DkMYB4 specifically recognize different MYB-binding cis-elements by electrophoretic mobility shift assays, arguing for certain degree of subfunctionalization ${ }^{28}$. On the other hand, a persimmon bHLH gene named DkMYC1 is underexpressed in non-astringent cultivars, following an expression pattern in fruit similar to $D k M Y B 4^{29}$. DkMYC1 protein interacts with DkMYB2 and DkMYB4, and these, in turn, interact with the WD40-repeat protein DkWDR1 by two-hybrid analysis, suggesting the conserved participation of MBW complexes in PA synthesis regulation in persimmon fruit ${ }^{26}$. In this study, we go further on the cytological and molecular characterization of MBW complex in persimmon by approaching the subcellular localization, protein interaction and transcriptional regulation effects of these MYB (DkMYB2 and DkMYB4), bHLH (DkMYC1) and WD40 (DkWDR1) components.

\section{Results}

Subcellular localization of MBW complex components. As transcription factors belonging to a hypothetical regulatory protein complex, DkMYB2, DkMYB4, DkMYC1 and DkWDR1 are expected to co-localize temporarily in the cell nucleus where they interact and perform their regulatory role within the framework of a developmental programme. The subcellular localization of these four putative MBW components in persimmon has been elucidated by transient expression in Nicotiana benthamiana leaves of the four corresponding genes fused to enhanced green fluorescent protein (eGFP) gene. According to the localization of specific nucleus and nucleolus markers, DkMYB2, DkMYB4 and DkMYC1 show differential nucleus/nucleolus partitioning when eGFP fusion is either at the N-terminal (Nt) or C-terminal (Ct), suggesting that eGFP position affects protein targeting (Fig. 1). Protein fusions with higher abundance in the nucleolus are DkMYB2-eGFP, DkMYB4-eGFP and eGFP-DkMYC1, whereas DkWDR1-eGFP shows appreciable presence in the cytoplasm in spite of its predominant localization in the nucleus. Overall, these four transcription factors show preferential localization in the nuclear compartment, and consequently physical interactions at the protein level occur most plausibly in the nucleus.

In vivo interaction of DkMYB2, DkMYB4, DkMYC1 and DkWDR1. Physical protein interactions among members of MBW complex involved in PAs accumulation in persimmon fruit has been only previously tested by the two-hybrid system in the yeast model, however no additional in planta evidences have been obtained on the formation of this complex. To asses this issue, we have assayed pair-wise interactions between DkMYB2, DkMYB4, DkMYC1 and DkWDR1 factors by bimolecular fluorescence complementation (BiFC) and transient expression in $\mathrm{N}$. benthamiana. The Nt and Ct fragments of the yellow fluorescent protein (YFP), required for reassembling of the fluorescent reporter, have been tested on both $\mathrm{Nt}$ and $\mathrm{Ct}$ sides of each transcription factor. Positive protein interactions by BiFC are shown in Fig. 2a. According to these results, DkMYB4 and DkMYC1 are able to interact with the rest of the factors and with themselves, and DkWDR1 does not reconstitute YFP fluorescence when paired with DkMYB2 and itself. This reproduces previous yeast two-hybrid results with few exceptions (Fig. 2b). Particularly, DkMYC1 homodimerization was not observed, and DkMYB2 self-interaction was not assayed due to autoactivation issues in former yeast-two hybrid experiments. Moreover, DkMYB2-DkWDR1 and DkMYC1-DkWDR1 interactions have been exclusively detected by yeast-two hybrid and BiFC analysis, respectively. A western analysis of transiently transformed leaves confirms that DkWDR1 and DkMYB2 were successfully co-expressed in different construct combinations and hence, the absent interaction of DkWDR1 with itself and DkMYB2 are not due to deficient protein synthesis or accumulation (Supplementary Fig. S1). Overall, these data support the biochemical ability of these factors to associate in a putative MBW complex in planta.

DkANR expression correlates well with PA accumulation and astringency. PA content has been measured at different points of fruit development in 'Hachiya' cultivar, starting in July and finishing in September, after external colour change has been initiated and before ripening leads to fruit softening and natural deastringency (Fig. 3a). During that period fruit average weight increases three-fold and the percentage of PA decreases concomitantly (Fig. 3a). Fruit soluble PA content is a balance between PA biosynthesis and insolubilization. 


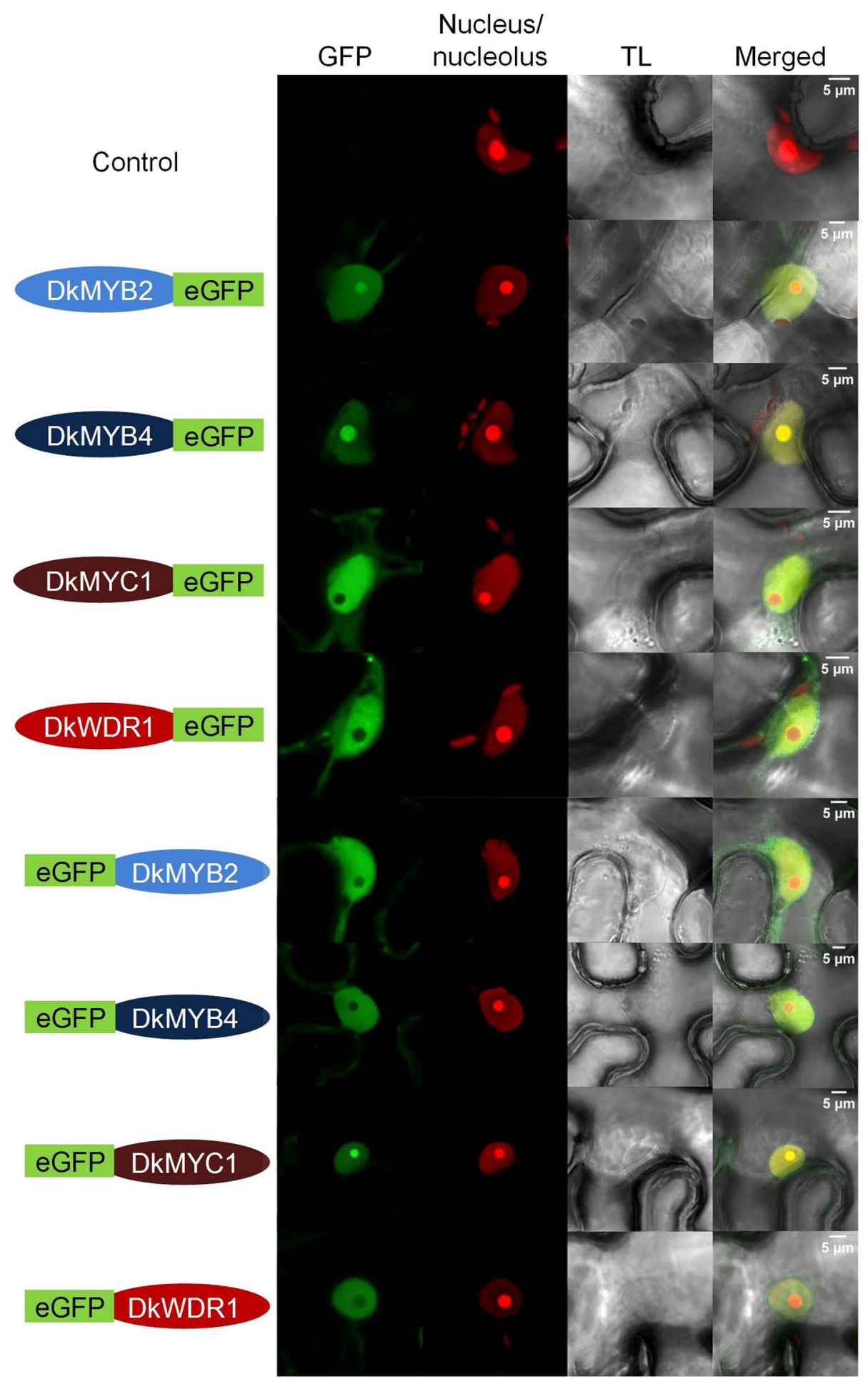

Figure 1. Nuclear localization of MBW factors. Nicotiana benthamiana leaves agroinfiltrated with DkMYB2, $D k M Y B 4, D k M Y C 1$ and DkWDR1 constructs containing Ct (right green label) or Nt (left green label) eGFP tags, were co-expressed with nucleus/nucleolus markers. The green (GFP), red (nucleus/nucleolus marker), transmitted light (TL) channels and merged images are shown in the figure. The fluorescent signals were visualized at 72 hours post-infiltration. Scale bars are shown in merged images.

PA insolubilization is mediated by acetaldehyde accumulation as a result of ripening and deastringency treatments ${ }^{10,11}$. Acetaldehyde content in these 'Hachiya' fruit samples reaches values around $0.1 \mathrm{mg}$ per $100 \mathrm{ml}$ of juice (Fig. 3b), which is by far lower than acetaldehyde produced in stored fruit and fruit treated for deastringency $y^{3,30}$. Thus, PA insolubilization due to acetaldehyde accumulation is not expected to contribute significantly to reduce 
a

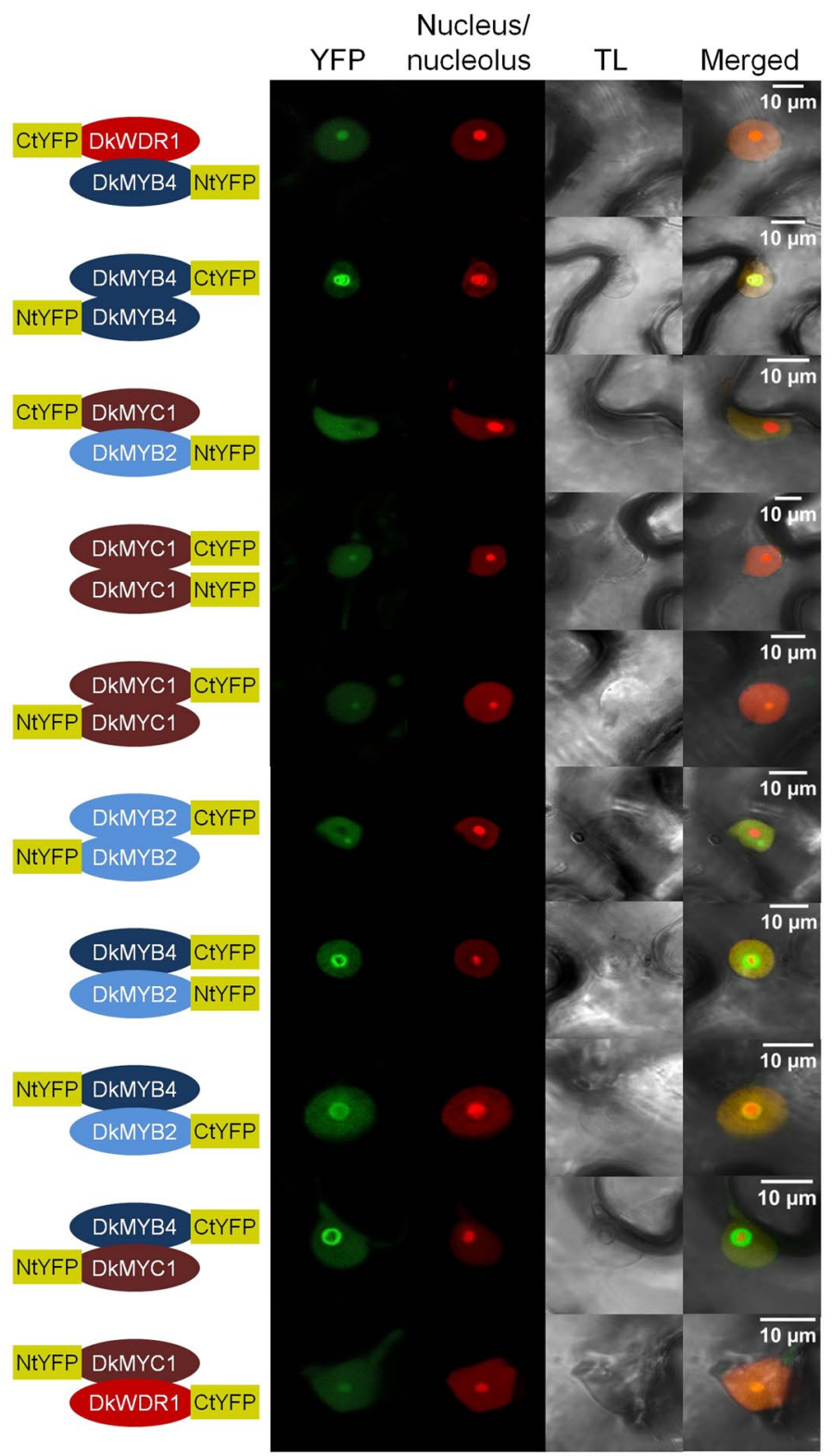

b

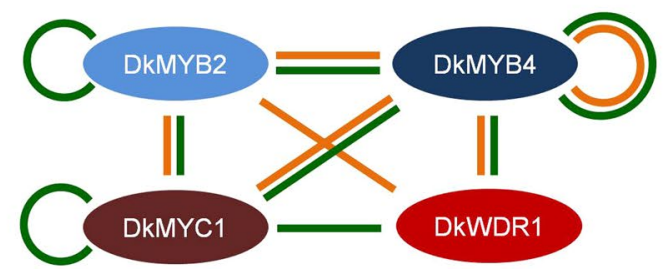

Figure 2. Protein interactions of MBW factors by bimolecular fluorescence complementation assays (BiFC). $N$. benthamiana leaves agroinfiltrated with different combinations of MBW factors fused to NtYFP or CtYFP peptides in Ct (right yellow label) or Nt (left yellow label) positions together with nucleus/nucleolus markers (a). The green reconstituted fluorescence (YFP), red (nucleus/nucleolus marker), transmitted light (TL) channels and merged images are shown in the figure. A diagram of previous yeast two-hybrid (orange lines) and positive BiFC interactions obtained in this study (green lines) (b). The fluorescent signals were visualized at 72 hours post-infiltration. Scale bars are shown in merged images.

soluble PA level in our samples, and PA content is mostly dependent on its biosynthesis rate. When representing the total estimated amount of PAs per fruit instead of its relative percentage, PA amount remains almost unchanged during the whole interval, with the exception of an initial increase in July samples (Fig. 3b). As we 


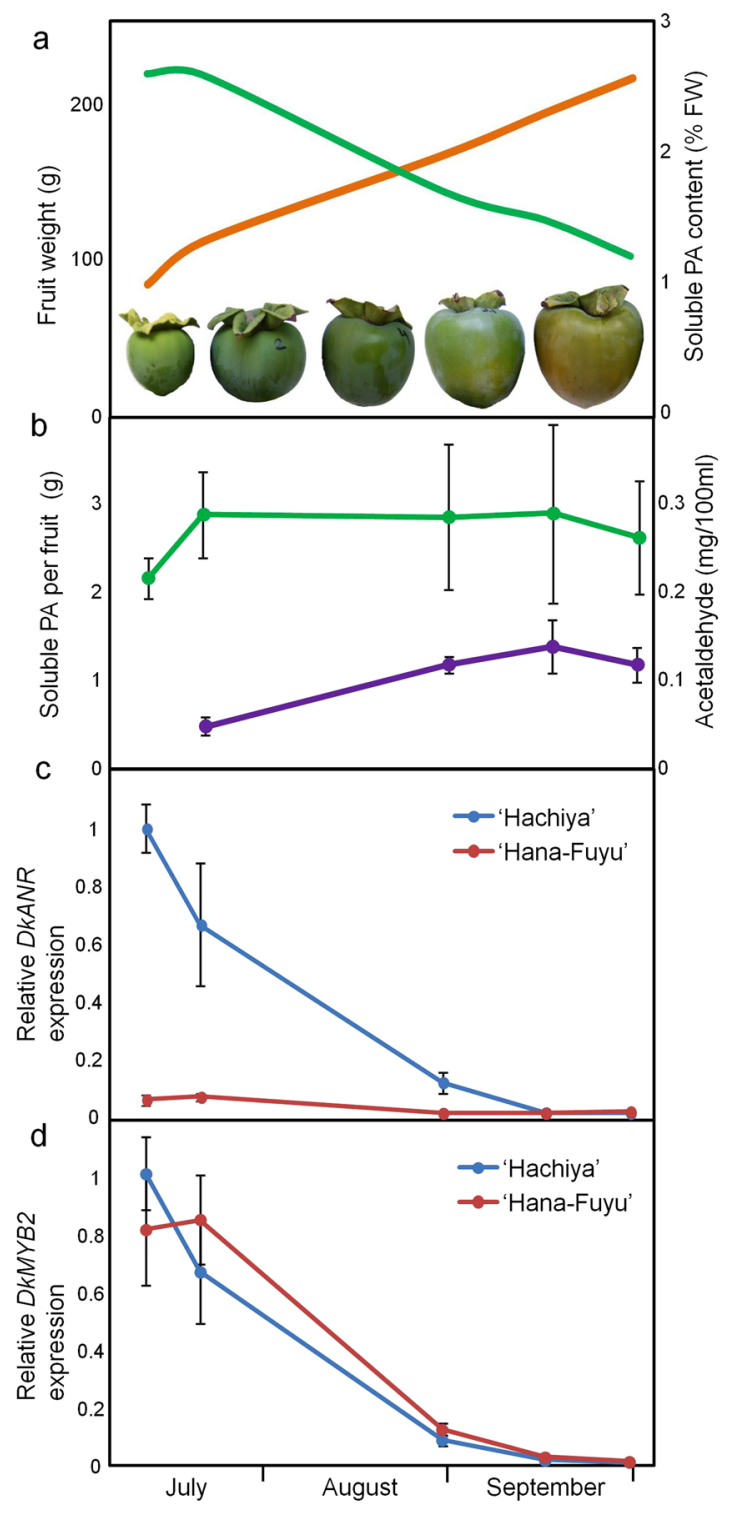

Figure 3. Proanthocyanidin (PA) content and gene expression during fruit development in persimmon. Fruit weight (orange line) and PA relative content (green line) in 'Hachiya' cultivar at different fruit development stages (a). Acetaldehyde accumulation (violet label) and total estimated PA content per fruit unit (green label) in 'Hachiya' (b). Relative gene expression of $D k A N R$ (c) and $D k M Y B 2$ (d) by qRT-PCR in the fruit samples shown in (a,b). The astringent cultivar 'Hachiya' (blue label) and the non-astringent cultivar 'Hana Fuyu' with the ast mutation (red label) have been analyzed. Data are means from four different fruits for anatomical and chemical analysis, and two biological replicates for gene expression. Error bars represent standard deviations.

consider that PA reduction by acetaldehyde-dependent insolubilization is relatively low, the observed decrease in relative PA content in Fig. 3a must be mostly due to a growth dilution effect, and the rate of PA biosynthesis is expected to be also low in this period.

As anthocyanidin reductase encoded by ANR gene has been postulated to perform a key role in PA biosynthesis, we have measured $D k A N R$ expression in 'Hachiya' fruit samples by qRT-PCR. DkANR transcript sharply decreases until a 0.01-fold change during the whole period (Fig. 3c), which cannot be explained by just growth dilution effects. On the contrary, it indicates a strong transcriptional repression in advanced developmental stages. The higher expression of $D k A N R$ in initial samples is in close agreement with the concurrent increase in total PA content per fruit (Fig. 3b). Then PA content remains steady in concordance with DkANR down-regulation (Fig. 3b,c). Such a positive correlation between $D k A N R$ expression and astringent-responsible PAs is confirmed in the low-PA non-astringent cultivar 'Hana Fuyu', which shows a constantly low DkANR expression during fruit development stages (Fig. 3c).

Other PA biosynthetic regulators, such as DkMYB4 and DkMYC1, reproduce well this low expression profile in 'Hana Fuyu' and other non-astringent cultivars, as shown in previous studies ${ }^{26,27,29}$. However, DkMYB2 relative expression decays in a similar way in both astringent and non-astringent cultivars (Fig. $3 \mathrm{~d}$ ), suggesting a common 
a

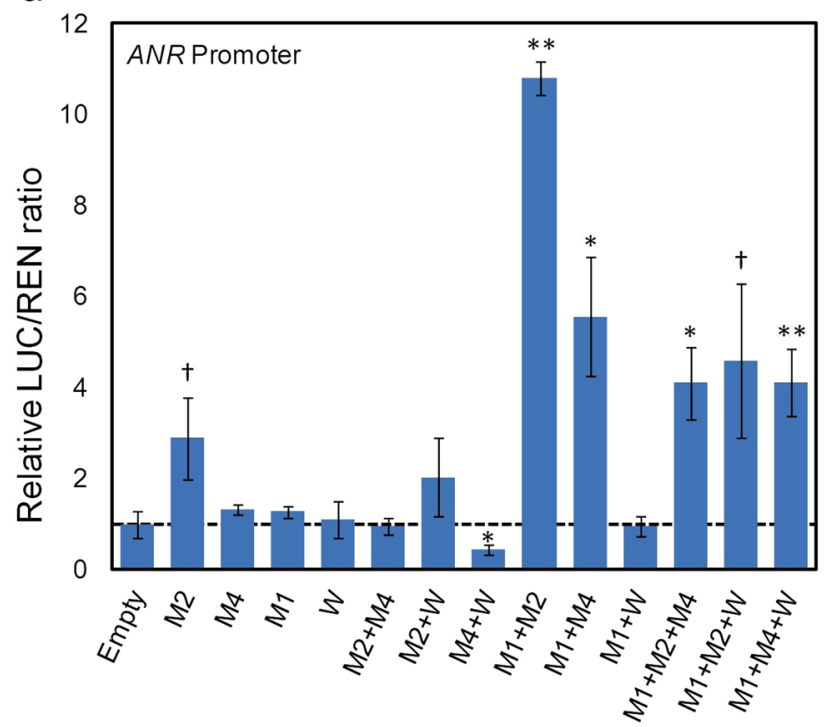

b

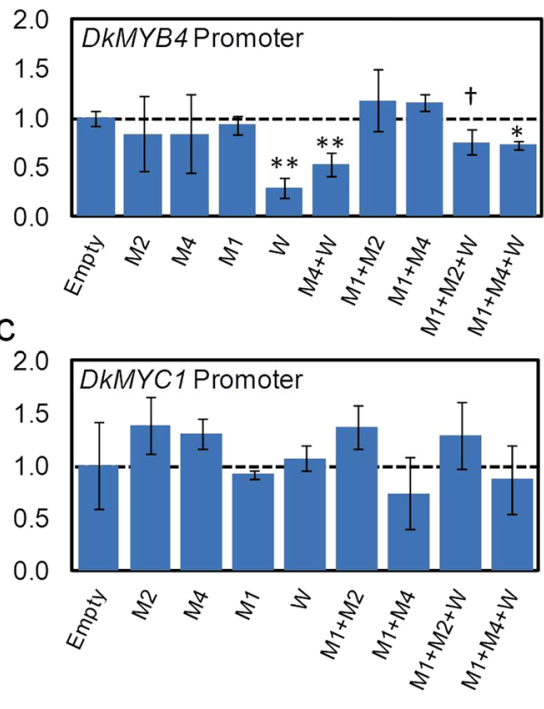

Figure 4. Analysis of the transcriptional activity of relevant PA biosynthesis regulatory promoters by dual luciferase assay. The LUC/REN ratio of $N$. benthamiana cells agroinfiltrated with $A N R(\mathbf{a}), D k M Y B 4(\mathbf{b})$ and $D k M Y C 1$ (c) promoters driving the LUC gene reporter, and regulatory genes DkMYB2 (M2), DkMYB4 (M4), $D k M Y C 1$ (M1) and DkWDR1 (W) under different combinations, was made relative to mock agroinfiltrations with pGreenII-62-SK (value of 1 labelled with a discontinuous line). Data are means from three replicates with error bars represent standard deviations. Symbols indicate statistical differences with respect to empty sample determined by Student's $t$ test $\left({ }^{\dagger} P<0.1, * P<0.05\right.$, ** $\left.P<0.01\right)$.

mechanism involving AST locus-dependent regulation of DkMYB4 and DkMYC1, with no impact on DkMYB2 expression.

Effect of MBW factors on the activity of ANR promoter. Based on PA-linked expression of these genes, we have cloned three DNA fragments (1.2-1.4kb) of the promoter and 5' UTR of DkMYB4, DkMYC1 and the $D$. lotus $A N R$, in the pGreenII-0800-LUC vector. These vectors synthesize luciferase reporter (LUC) under the action of our selected promoters, in order to test the regulatory effect of individual and combined MBW factors on the activity of these promoters by a dual luciferase assay, using expression of the REN reporter gene as internal reference.

$D k M Y B 2$ transient expression in $N$. benthamiana leaves increases ANR promoter transcriptional activity three-fold, whereas DkMYB4, DkMYC1 and DkWDR1 do not modify it significantly (Fig. 4a). Interestingly, any combination of two or three elements containing both $D k M Y C 1$ and a MYB gene (DkMYB2 or DkMYB4), strongly increases LUC/REN ratio, being highest when $D k M Y C 1$ and $D k M Y B 2$ are co-expressed. On the contrary, $D k W D R 1$ does not improve $A N R$ promoter expression under any gene combination.

As DkMYB4 and DkMYC1 genes are similarly down-regulated during fruit development concomitantly with $P A$ reduction, and are differentially expressed in a non-astringent cultivar ${ }^{26,27,29}$, their promoters have been also cloned and tested by dual luciferase assays in $N$. benthamiana. MBW factors assayed in the experiment do not increase the activity of DkMYB4 nor DkMYC1 promoters, however DkWDR1 transient expression associates with a significant decrease in LUC expression driven by DkMYB4 promoter (Fig. 4b,c).

\section{Discussion}

DkMYB2, DkMYB4, DkMYC1 and DkWDR1 have been postulated to co-regulate the expression of PA biosynthesis genes in persimmon as a complex ${ }^{26}$, and hence a coordinated nuclear co-localization of them is expected. Related components of MBW complexes in other species have been found located in the nucleus (TT2) ${ }^{31}$, or partitioned in nucleus and cytoplasm (TTG1 and VvMYC1) ${ }^{23,32}$. In this study, DkMYB2, DkMYB4, DkMYC1 and DkWDR1 have been mostly localized in the cell nucleus, but DkMYB2, DkMYB4, DkMYC1 show a differential nucleus-nucleolus partitioning depending on the $\mathrm{Nt}$ or $\mathrm{Ct}$ position of the eGFP fusion. To our knowledge, this has not been previously observed in other MBW factors, although in most of these cases, only one Nt or Ct fusion is assayed. Indeed, a systematic approach in yeast has shown that a high percentage of proteins display different subcellular localization when GFP is tagged at either the $\mathrm{Nt}$ or $\mathrm{Ct}^{33}$.

Transient expression in N. benthamiana and BiFC analysis support the ability of DkMYB2, DkMYB4, DkMYC1 and DkWDR1 proteins to interact with each other in vivo, which in fact reinforces previous two-hybrid data in the yeast Saccharomyces cerevisiae ${ }^{26}$. The only combinatorial interactions not confirmed by BiFC are DkMYB2-DkWDR1 and DkWDR1 with itself. DkMYB2-DkWDR1 interaction was observed in a previous two-hybrid study, thus only homodimerization of DkWDR1 is not sustained on experimental evidences. These $\mathrm{BiFC}$ and yeast two-hybrid interaction data are compatible with a multitude of possible combinations and sizes 


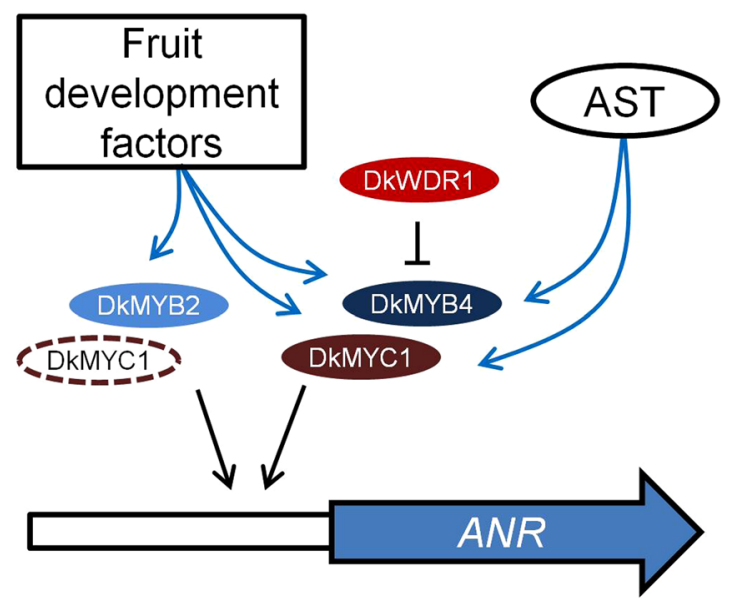

Figure 5. Diagram of regulatory factors affecting $A N R$ expression. $A N R$ coding gene is represented as a wide blue arrow, and its promoter as a contiguous white rectangle. Regulatory proteins are elliptic forms. The discontinuous ellipse of DkMYC1 indicates it is dispensable for the positive effect of DkMYB2. The transcriptional effect is labelled as a black arrow (inductive) or a black line ended in a perpendicular bar (repressive). Fruit development-dependent factors and AST locus modify the expression of MYB and bHLH factors by a yet unknown mechanism (blue arrows).

of the complex, which presumably enable a high degree of functional and regulatory versatility. Physical interactions among MBW factors involved in PA production have been also verified in Arabidopsis ${ }^{20}$, grapevine ${ }^{23}$, strawberry ${ }^{24}$, and tea plant ${ }^{34}$ among other PA and flavonoid biosynthesis complexes.

These BiFC and subcellular localization results strongly support the formation in vivo of protein complexes comprising at least several of these factors, but conclusive functional evidences about their coordinated recruitment to modify the expression of PA biosynthetic genes in persimmon fruit are scarce. Anthocyanidin reductase is the main enzyme specifically involved in PA production in persimmon, and its coding ANR gene is considered a major target of transcriptional regulation ${ }^{19}$, being consequently a proper candidate gene for studying PA biosynthesis regulation. Previous dual luciferase assays in N. benthamiana have shown that DkMYB2 and DkMYB4 increase the activity of ANR promoter when co-expressed with Arabidopsis AtEGL3 gene coding for a bHLH protein involved in regulation of the flavonoid pathway, but not when expressed individually ${ }^{28}$. On the contrary, in our hands, $D k M Y B 2$ is able to increase $A N R$ promoter activity three-fold in the absence of other factors, which suggests that DkMYB2 does not require a complete MBW complex to enhance, at some level, ANR expression and consequently improve PA production (Fig. 5). Interestingly, the ectopic expression of DkMYB2 in kiwifruit calluses induces PA accumulation without additional MBW components ${ }^{28}$. DkMYB2 expression is regulated by fruit maturation factors in persimmon that markedly reduce it in advanced stages of development, similarly to $A N R, D k M Y B 4$ and $D k M Y C 1$, but in contrast to these genes it seems not to be impaired in ast non-astringent mutants. Unexpectedly, DkMYB2 has only a minor contribution to PA accumulation in these mutants, which is most likely due to its low expression level in fruit in comparison with $D k M Y B 4^{27}$.

We have employed for the first time the persimmon bHLH (DkMYC1) and WD40 (DkWDR1) components in transient expression assays in combination with MYB factors, for the elucidation of the function of MBW complexes in the expression of PA responsive genes in persimmon fruit. DkMYC1 does not affect significantly ANR expression on its own, but consistently intensifies the effect of DkMYB2 and DkMYB4. In Arabidopsis, there is a similar synergistic effect of TT2 (MYB) and TT8 (bHLH) on the expression of $B A N$ that responds to a stronger cooperative binding of the pair TT2-TT8 to BAN promoter ${ }^{20}$. Thus, the low expression of $A N R$ gene from early stages of fruit development in the ast non-astringent cultivar 'Hana Fuyu' (Fig. 3c) seems to be caused by the concomitant defective expression of DkMYB4 and DkMYC1 genes in this mutant ${ }^{26}$ (Fig. 5). Contrarily to the positive effect of TTG1 (WD40) gene overexpression, and the negative effect of TTG1 silencing on BAN expression in Arabidopsis ${ }^{20}, D k W D R 1$ expression does not affect $A N R$ promoter activity in our transient expression experiments. The strawberry ortholog of TTG1 also increases Arabidopsis BAN expression in combination with its bHLH and MYB co-interactors ${ }^{24}$, which suggests the presence of certain functional particularities in persimmon DkWDR1 or perhaps regulatory differences between ANR and BAN promoters. On the other side, WD40 proteins act as structural platforms for facilitating protein-protein interaction, and consequently its effect on the positive transcriptional activity of the complex could be shaded by the ectopic overexpression of components of the complex and the presence of endogenous similar factors in N. benthamiana cells.

As DkMYB4 and DkMYC1 show a development and cultivar dependent expression profile highly similar to $A N R^{26,27,29}$ (Fig. 3c), we considered plausible a self-regulatory loop in the expression of these genes, similar to the positive feedback mechanism operating in TT8 from Arabidopsis ${ }^{35}$. However, DkMYB4 and DkMYC1 promoters are not activated by any MBW element utilized in this study. On the contrary, DkWDR1 reduces the expression of DkMYB4 by itself and in combination with MYB genes. This repressive effect of DkWDR1 resembles the activity of MBW complexes containing MYB proteins showing at the C-terminal end an ethylene-responsive element binding factor-associated amphiphilic repression (EAR) motif ${ }^{36}$. Thus, binding of specific repressive MYB 
proteins has potentially the ability to turn MBW complex into a repressive factor. In light of these data we cannot discard a repressive role of $D k W D R 1$ on the expression of $D k M Y B 4$ and other genes, which could depend on the binding of distinct MYB of bHLH elements with specific activating or repressive domains.

\section{Methods}

Plant material. Diospyros kaki Thunb. cvs 'Hachiya' (astringent fruit) and 'Hana Fuyu' (non-astringent fruit) were grown in an orchard located in Museros (Spain; $39^{\circ} 34^{\prime} 40^{\prime \prime} \mathrm{N}, 0^{\circ} 21^{\prime} 46^{\prime \prime} \mathrm{W}$ ) under standard agricultural practices $^{37}$. Four fruit samples per cultivar were collected at different maturation stages on the following dates in 2011: July 12, July 21, August 30, September 16 and September $30^{26}$. Soluble tannins were evaluated using the Folin-Denis method ${ }^{38}$, as described previously ${ }^{39}$, and results were expressed as percentage of fresh weight (FW).

Gene isolation. The Diospyros kaki (cv. 'Hachiya') genes DkMYB2 (AB503699.1), DkMYB4 (KR057233.1), DkMYC1 (KR057234.1) and DkWDR1 (KR057229.1) were obtained from pGADT7 plasmids described in a previous study ${ }^{26}$. In order to identify $D k M Y B 4$ and $D k M Y C 1$ promoter sequences, a manual assembly of $D$. lotus cv. Kunsenshi genome reads stored in the Sequence Read Archive (SRA) database (ID: SRP045872) was performed ${ }^{40}$. For amplifying $A N R$ promoter, we designed primers (Supplementary Table S1) from the previously published sequence of $D$. lotus gene (AB504523.1). ANR promoter amplification was not possible in $D$. kaki, and therefore the D. lotus promoter was used in the analysis. $D k M Y B 4$ and $D k M Y C 1$ promoters were amplified from $D$. kaki cv. 'Hachiya' genomic DNA (Supplementary Table S1). The DNA of both D. kaki and D. lotus was extracted from fresh leaves following a standard CTAB DNA extraction protocol ${ }^{41}$.

Plasmid construction. For the construction of subcellular localization and BiFC vectors, each of the four genes were amplified (Supplementary Table S1) with a $15 \mathrm{bp}$ target vector residue at the $5^{\prime}$ end needed for recombination with the In-Fusion HD Cloning kit (TAKARA BIO, Otsu, Japan). Fragments were purified and cloned into ampicillin resistant pSK +35 S-eGFP-PoPit vectors ${ }^{42}$ and two cassettes for each protein were made for subcellular localization. Four cassettes for each protein were made with ampicillin resistant pSK $+35 \mathrm{~S}-(\mathrm{N}-\mathrm{YFP}$ or $\mathrm{C}$-YFP)-PoPit vector ${ }^{43}$ for BiFC analysis. The fragments containing the expression cassette from pSK vectors were digested with HindIII and subcloned into the kanamycin resistant pMOG800 vector ${ }^{44}$.

In order to construct the vectors for transient expression, the four genes contained in pGADT7 plasmids ${ }^{26}$ were digested with $\mathrm{SacI}$ and XhoI to release the insert. The fragments were purified and inserted in a pGreenII62 -SK kanamycin resistant vector ${ }^{45}$, previously linearized with SacI/XhoI. The promoter sequences were amplified from vectors using specific primers with restriction enzyme sequences tails at $5^{\prime}$ (Supplementary Table S1). The purified PCR products were digested (HindIII/PstI for ANR and DkMYC1 promoters and HindIII/NcoI for $D k M Y B 4$ promoter), purified and cloned into linearized pGreenII-0800-LUC kanamycin resistant vector ${ }^{45}$.

All the described vectors were provided by Dr. J. A. Sánchez-Navarro (Instituto de Biología Molecular y Celular de Plantas "Primo Yúfera", Valencia, Spain). Plasmids containing gene and promoter constructs were finally transferred to Agrobacterium tumefaciens strain C58 by electroporation. For pMOG800 vectors, transformation was carried out in bacteria containing the virulence helper plasmid pCH32 $2^{46}$. All DNA constructions were verified by plasmid DNA sequencing.

Subcellular localization of MBW complex proteins in vivo. To characterize the subcellular localization of the MBW complex components, each protein was fused at either the Nt or the Ct of eGFP and transiently expressed in planta. For a better visualization of the fluorescence signal, all proteins were co-expressed with the silencing suppressor HC-Pro protein from the Tobacco Etch Virus ${ }^{43}$. A. tumefaciens C58 strains were grown overnight in LB media supplemented with kanamycin and rifampicin, at $28^{\circ} \mathrm{C}$. Cultures were centrifuged $5 \mathrm{~min}$ at $4,000 \times \mathrm{g}$, and pellets were resuspended in infiltration media $\left(\mathrm{MgCl}_{2} 10 \mathrm{mM}+\mathrm{MES} 10 \mathrm{mM} \mathrm{pH} 5.6\right)$ to an $\mathrm{OD}_{600}$ of 0.5 for each construct and an $\mathrm{OD}_{600}$ of 0.1 for the HC-Pro. N. benthamiana young plants (2 pairs of leaves) were agroinfiltrated as previously described ${ }^{47}$. Plants remained in a greenhouse at $24^{\circ} \mathrm{C}$ (day) and $18^{\circ} \mathrm{C}$ (night) with a $16 \mathrm{~h}$ light photoperiod. Three days after infiltration, leaf samples were collected and mounted in a microscope slide with a drop of water. Observation of the fluorescence in the underside epidermis was performed with a LEICA TCS SL confocal microscope $\left(\lambda_{\mathrm{exc}}=488 \mathrm{~nm} ; \lambda \mathrm{e}_{\mathrm{m}}=492-533 \mathrm{~nm}\right.$ for eGFP). For the nucleus and nucleolus subcellular colocalization, the proteins were coinfiltrated with cultures $\left(\mathrm{OD}_{600} 0.1\right)$ expressing the NLS of SV40 large $\mathrm{T}$ antigen fused to the red fluorescent protein and the fibrillarin fused to the cherry fluorescent protein, respectively $\left(\lambda_{\text {exc }}=561 \mathrm{~nm} ; \lambda \mathrm{e}_{\mathrm{m}}=588-634 \mathrm{~nm}\right)$.

Bimolecular fluorescence complementation assays (BiFC). In the BiFC assay ${ }^{48}$, addressed to characterize the interaction between the components of the hypothetical MBW complex, all the possible two-by-two combinations of homodimers and heterodimers were assayed in planta. Chimeric proteins were transiently co-expressed in $N$. benthamiana using $A$. tumefaciens (strain C58) cultures $\left(\mathrm{OD}_{600}=0.4\right)$ transformed with the corresponding binary plasmids pMOG800, as previously described ${ }^{47}$. To increase the expression of the different proteins, we included an $A$. tumefaciens culture $\left(\mathrm{OD}_{600}=0.1\right)$ expressing the HC-Pro. At 3 days post-infiltration, the fluorescence reconstitution was monitored in the confocal LEICA TCS SL $\left(\lambda_{\mathrm{exc}}=488 \mathrm{~nm} ; \lambda_{\mathrm{em}}=492-533 \mathrm{~nm}\right)$.

Western blot assay. Samples from the BiFC assay were immediately frozen in liquid nitrogen. Frozen leaves $\left(50 \mathrm{mg}\right.$ ) were ground and proteins were extracted with $200 \mu \mathrm{l}$ of Laemmli buffer ${ }^{49}$, boiled for 5 min and centrifuged for $1 \mathrm{~min}$ at $15,800 \times \mathrm{g}$ to pellet cellular debris. For protein separation, $25 \mu \mathrm{l}$ of the mixture was subjected to $12 \%$ sodium dodecylsulphate-polyacrylamide gel electrophoresis (SDS-PAGE). Gels were electrotransferred to nitrocellulose membranes following the manufacturer's recommendations. Proteins were detected on western blots using an anti-GFP N-terminal antibody (SIGMA, St. Louis, MO, USA; cat. no. G1544) for N-terminal yellow fluorescent protein (NtYFP) fusions, and anti-GFP antibody (ROCHE, Basel, Switzerland; cat. no. 11814460001) 
for C-terminal YFP (CtYFP) fusions, followed by a secondary peroxidase-labelled antibody and incubation with a chemiluminescence substrate (AMERSHAM, ECLTM Prime Western Blotting Detection Reagent). The chemiluminescence was detected exposing photographic film to the membranes.

Dual luciferase assay. To determine the effects of the hypothetical MBW protein complex on the promoters of $A N R, D k M Y B 4$ and $D k M Y C 1$ genes, a dual luciferase assay was performed. The effect of the homo and heterodimers formed by two and three proteins was assayed by the co-expression of the promoter and protein vectors as previously described in N. benthamiana with A. tumefaciens strain C58. HC-Pro was also used for enhancing the transient expression of the different proteins. After 3 days, $30 \mathrm{mg}$ of agroinfiltrated leaves from 3 biological replicates, were sampled for each combination. Samples were immediately frozen in liquid nitrogen. For measuring promoter activity, samples were ground to powder and then Firefly (LUC) and Renilla (REN) luciferase activity was measured following the Dual-Luciferase Reporter Assay System (PROMEGA, Madison, WI, USA) with the aid of a PROMEGA GloMax Multi Microplate Reader luminometer. Promoter activity was measured as the quotient between the LUC/REN ratio of promoter plus transcription factors samples and the $\mathrm{LUC/REN} \mathrm{ratio} \mathrm{of} \mathrm{promoter} \mathrm{without} \mathrm{additional} \mathrm{factors.}$

Isolation of RNA and quantitative real-time RT-PCR (qRT-PCR). Total RNA was isolated from $150 \mathrm{mg}$ of fruit flesh using a cetyltrimethylammonium bromide (CTAB)-based procedure ${ }^{50}$. Genomic DNA was removed with the RNase-Free DNase Set (QIAGEN, Hilden, Germany) according to manufacturer's instructions. Purified RNA was reverse transcribed with PrimeScript RT Reagent Kit (TAKARA BIO). qRT-PCR was performed in a StepOnePlus Real-Time PCR System (LIFE TECHNOLOGIES, Carlsbad, CA, USA), using 1-2 $\mu$ of 10X diluted cDNA, SYBR premix Ex Taq (Tli RNaseH plus) (TAKARA BIO) and primers shown in Supplementary Table S1, in a total volume of $20 \mu \mathrm{l}$. The PCR protocol consisted of $10 \mathrm{~min}$ at $95^{\circ} \mathrm{C}$, followed by 40 cycles of $15 \mathrm{~s}$ at $95^{\circ} \mathrm{C}$, and $1 \mathrm{~min}$ at $60^{\circ} \mathrm{C}$. PCR specificity was confirmed by the presence of a single peak in the dissociation curve and by agarose electrophoresis. We used DkActin as reference gene $\mathrm{e}^{26,27}$. A relative standard curve procedure was employed for measuring relative expression. Results were the average of two independent biological replicates with $2-3$ technical replicates each.

Received: 5 October 2019; Accepted: 13 February 2020;

Published online: 26 February 2020

\section{References}

1. Dixon, R. A., Xie, D.-Y. \& Sharma, S. B. Proanthocyanidins-a final frontier in flavonoid research? New Phytol. 165, 9-28 (2005).

2. Yonemori, K. \& Matsushima, J. Property of development of the tannin cells in non-astringent type fruits of Japanese persimmon (Diospyros kaki) and its relationship to natural deastringency. J. Jpn. Soc. Hortic. Sci. 54, 201-208 (1985).

3. Salvador, A. et al. Physiological and structural changes during ripening and deastringency treatment of persimmon fruit cv. 'Rojo Brillante'. Postharvest Biol. Tec. 46, 181-188 (2007).

4. Bernays, E. A., Driver, G. C. \& Bilgener, M. Herbivores and plant tannins. In Advances in Ecological Research (eds. Begon, M., Fitter, A. H., Ford, E. D. \& MacFadyen, A.) 19, 263-302 (Academic Press, 1989).

5. Tessmer, M. A. et al. Microstructural changes while persimmon fruits mature and ripen. Comparison between astringent and nonastringent cultivars. Postharvest Biol. Tec. 120, 52-60 (2016).

6. Nishiyama, S. et al. Characterization of a gene regulatory network underlying astringency loss in persimmon fruit. Planta 247, 733-743 (2018).

7. Sugiura, A., Yonemori, K., Harada, H. \& Tomama, T. Changes of ethanol and acetaldehyde contents in Japanese persimmon fruits and their relation to natural deastringency. Studies from Inst. Hort. Kyoto Univ. 9, 41-47 (1979).

8. Sugiura, A. \& Tomana, T. Relationships of ethanol production by seeds of different types of Japanese persimmons and their tannin content. HortSci. 18, 319-321 (1983).

9. Ben-Arie, R. \& Sonego, L. Temperature affects astringency removal and recurrence in persimmon. J. Food Sci. 58, 1397-1400 (1993).

10. Matsuo, T. \& Itoo, S. A model experiment for de-astringency of persimmon fruit with high carbon dioxide treatment: in vitro gelation of kaki-tannin by reacting with acetaldehyde. Agr. Biol. Chem. Tokyo 46, 683-689 (1982).

11. Pesis, E. \& Ben-Arie, R. Involvement of acetaldehyde and ethanol accumulation during induced deastringency of persimmon fruits. J. Food Sci. 49, 896-899 (1984).

12. Kanzaki, S., Yonemori, K., Sugiura, A., Sato, A. \& Yamada, M. Identification of molecular markers linked to the trait of natural astringency loss of Japanese persimmon (Diospyros kaki) fruit. J. Am. Soc. Hortic. Sci. 126, 51-55 (2001).

13. Yamada, M. \& Sato, A. Segregation for fruit astringency type in progenies derived from crosses of 'Nishimurawase' $\times$ pollination constant non-astringent genotypes in oriental persimmon (Diospyros kaki Thunb.). Sci. Hortic.-Amsterdam 92, 107-111 (2002).

14. Besada, C. et al. Chloride stress triggers maturation and negatively affects the postharvest quality of persimmon fruit. Involvement of calyx ethylene production. Plant Physiol. Biochem. 100, 105-112 (2016).

15. Lepiniec, L. et al. Genetics and biochemistry of seed flavonoids. Annu. Rev. Plant Biol. 57, 405-430 (2006).

16. Tanner, G. J. et al. Proanthocyanidin biosynthesis in plants. Purification of legume leucoanthocyanidin reductase and molecular cloning of its cDNA. J. Biol. Chem. 278, 31647-31656 (2003).

17. Xie, D.-Y., Sharma, S. B., Paiva, N. L., Ferreira, D. \& Dixon, R. A. Role of anthocyanidin reductase, encoded by BANYULS in plant flavonoid biosynthesis. Science 299, 396-399 (2003).

18. Ikegami, A., Eguchi, S., Kitajima, A., Inoue, K. \& Yonemori, K. Identification of genes involved in proanthocyanidin biosynthesis of persimmon (Diospyros kaki) fruit. Plant Science 172, 1037-1047 (2007).

19. Akagi, T. et al. Expression balances of structural genes in shikimate and flavonoid biosynthesis cause a difference in proanthocyanidin accumulation in persimmon (Diospyros kaki Thunb.) fruit. Planta 230, 899-915 (2009).

20. Baudry, A. et al. TT2, TT8, and TTG1 synergistically specify the expression of BANYULS and proanthocyanidin biosynthesis in Arabidopsis thaliana. Plant J. 39, 366-380 (2004).

21. Xu, W. et al. Complexity and robustness of the flavonoid transcriptional regulatory network revealed by comprehensive analyses of MYB-bHLH-WDR complexes and their targets in Arabidopsis seed. New Phytol. 202, 132-144 (2014).

22. Xu, W., Dubos, C. \& Lepiniec, L. Transcriptional control of flavonoid biosynthesis by MYB-bHLH-WDR complexes. Trends Plant Sci. 20, 176-185 (2015)

23. Hichri, I. et al. The basic helix-loop-helix transcription factor MYC1 is involved in the regulation of the flavonoid biosynthesis pathway in grapevine. Mol. Plant 3, 509-523 (2010). 
24. Schaart, J. G. et al. Identification and characterization of MYB-bHLH-WD40 regulatory complexes controlling proanthocyanidin biosynthesis in strawberry (Fragaria $\times$ ananassa) fruits. New Phytol. 197, 454-467 (2013).

25. Gesell, A., Yoshida, K., Tran, L. T. \& Constabel, C. P. Characterization of an apple TT2-type R2R3 MYB transcription factor functionally similar to the poplar proanthocyanidin regulator PtMYB134. Planta 240, 497-511 (2014).

26. Naval, M. et al. A WD40-repeat protein from persimmon interacts with the regulators of proanthocyanidin biosynthesis DkMYB2 and DkMYB4. Tree Genet. Genomes 12, 13 (2016).

27. Akagi, T. et al. DkMyb4 is a Myb transcription factor involved in proanthocyanidin biosynthesis in persimmon fruit. Plant Physiol. 151, 2028-2045 (2009).

28. Akagi, T., Ikegami, A. \& Yonemori, K. DkMyb2 wound-induced transcription factor of persimmon (Diospyros kaki Thunb.), contributes to proanthocyanidin regulation. Planta 232, 1045-1059 (2010).

29. Su, F., Hu, J., Zhang, Q. \& Luo, Z. Isolation and characterization of a basic Helix-Loop-Helix transcription factor gene potentially involved in proanthocyanidin biosynthesis regulation in persimmon (Diospyros kaki Thunb.). Sci. Hortic.-Amsterdam 136, 115-121 (2012).

30. Hribal, J., Zavrtanik, M., Simćić, M. \& Vidrih, R. Changes during storing and astringency removal of persimmon fruit Diospyros kaki L. Acta Aliment. Hung. 29, 123-136 (2000).

31. Nesi, N., Jond, C., Debeaujon, I., Caboche, M. \& Lepiniec, L. The Arabidopsis TT2 gene encodes an R2R3 MYB domain protein that acts as a key determinant for proanthocyanidin accumulation in developing seed. Plant Cell 13, 2099-2114 (2001).

32. Zhao, M., Morohashi, K., Hatlestad, G., Grotewold, E. \& Lloyd, A. The TTG1-bHLH-MYB complex controls trichome cell fate and patterning through direct targeting of regulatory loci. Development 135, 1991-1999 (2008).

33. Weill, U. et al. Assessment of GFP tag position on protein localization and growth Fitness in yeast. J. Mol. Biol. 431, 636-641 (2019).

34. Wang, P. et al. A sucrose-induced MYB (SIMYB) transcription factor promoting proanthocyanidin accumulation in the tea plant (Camellia sinensis). J. Agric. Food Chem. 67, 1418-1428 (2019).

35. Baudry, A., Caboche, M. \& Lepiniec, L. TT8 controls its own expression in a feedback regulation involving TTG1 and homologous MYB and bHLH factors, allowing a strong and cell-specific accumulation of flavonoids in Arabidopsis thaliana. Plant J. 46, 768-779 (2006).

36. Albert, N. W. et al. A conserved network of transcriptional activators and repressors regulates anthocyanin pigmentation in eudicots. Plant Cell 26, 962-980 (2014).

37. Bellini, E. Cultural practices for persimmon production. In Options Méditerranéennes. Série A: Séminaires Méditerranéens (CIHEAM) (eds. Bellini, E. \& Giordani, E.) 51. 39-52 (CIHEAM-IAMZ, 2002).

38. Taira, S. Astringency in Persimmon. In Fruit Analysis (eds. Linskens, H. F. \& Jackson, J. F.) 97-110 (Springer Berlin Heidelberg, 1995).

39. Arnal, L. \& Rio, M. A. D. Quality of persimmon fruit cv. Rojo brillante during storage at different temperatures. Span. J. Agric. Res. 2, 243-247 (2004).

40. Akagi, T., Henry, I. M., Tao, R. \& Comai, L. A Y-chromosome-encoded small RNA acts as a sex determinant in persimmons. Science 346, 646-650 (2014).

41. Doyle, J. J. \& Doyle, J. L. A rapid DNA isolation procedure for small quantities of fresh leaf tissue. Phytochemical Bulletin 19, 11-15 (1987).

42. Herranz, M. C., Sanchez-Navarro, J. A., Aparicio, F. \& Pallás, V. Simultaneous detection of six stone fruit viruses by non-isotopic molecular hybridization using a unique riboprobe or 'polyprobe'. J. Virol. Methods 124, 49-55 (2005).

43. Leastro, M. O., Pallás, V., Resende, R. O. \& Sánchez-Navarro, J. A. The movement proteins (NSm) of distinct tospoviruses peripherally associate with cellular membranes and interact with homologous and heterologous NSm and nucleocapsid proteins. Virology 478, 39-49 (2015).

44. Knoester, M. et al. Ethylene-insensitive tobacco lacks nonhost resistance against soil-borne fungi. Proc. Natl. Acad. Sci. USA 95, 1933-1937 (1998).

45. Hellens, R. P., Edwards, E. A., Leyland, N. R., Bean, S. \& Mullineaux, P. M. pGreen: a versatile and flexible binary Ti vector for Agrobacterium-mediated plant transformation. Plant Mol. Biol. 42, 819-832 (2000).

46. Hamilton, C. M., Frary, A., Lewis, C. \& Tanksley, S. D. Stable transfer of intact high molecular weight DNA into plant chromosomes. Proc. Natl. Acad. Sci. USA 93, 9975-9979 (1996).

47. Genovés, A., Pallás, V. \& Navarro, J. A. Contribution of topology determinants of a viral movement protein to its membrane association, intracellular traffic, and viral cell-to-cell movement. J. Virol. 85, 7797-7809 (2011).

48. Aparicio, F., Sánchez-Navarro, J. A. \& Pallás, V. In vitro and in vivo mapping of the Prunus necrotic ringspot virus coat protein C-terminal dimerization domain by bimolecular fluorescence complementation. J. Gen. Virol. 87, 1745-1750 (2006)

49. Laemmli, U. K. Cleavage of structural proteins during the assembly of the head of bacteriophage T4. Nature 227, 680-685 (1970).

50. Gambino, G., Perrone, I. \& Gribaudo, I. A Rapid and effective method for RNA extraction from different tissues of grapevine and other woody plants. Phytochem. Anal. 19, 520-525 (2008).

\section{Acknowledgements}

This work was funded by the Instituto Nacional de Investigación y Tecnología Agraria y Alimentaria (INIA)FEDER (grant no. RF2013-00043-C02-02 and RTA2017-00011-C03-01). FG-M was funded by a fellowship cofinanced by the Generalitat Valenciana and European Social Fund (2014-2020) (grant no. ACIF/2016/115). We are grateful to Sara Selma for providing scientific and technical advice.

\section{Author contributions}

J.A.S.N., M.L.B., M.M.N. and G.R. contributed to the experimental design. F.G.M. and M.M.N. made gene isolation and cloning. F.G.M. performed protein localization, BiFC, qRT-PCR, dual luciferase and western blot experiments. C.B. and A.S. performed fruit measurements. F.G.M., J.A.S.N., M.M.N. and G.R. analyzed the data. G.R. wrote the paper. All authors reviewed and approved the manuscript.

\section{Competing interests}

The authors declare no competing interests.

\section{Additional information}

Supplementary information is available for this paper at https://doi.org/10.1038/s41598-020-60635-w.

Correspondence and requests for materials should be addressed to G.R.

Reprints and permissions information is available at www.nature.com/reprints. 
Publisher's note Springer Nature remains neutral with regard to jurisdictional claims in published maps and institutional affiliations.

(c) (i) Open Access This article is licensed under a Creative Commons Attribution 4.0 International License, which permits use, sharing, adaptation, distribution and reproduction in any medium or format, as long as you give appropriate credit to the original author(s) and the source, provide a link to the Creative Commons license, and indicate if changes were made. The images or other third party material in this article are included in the article's Creative Commons license, unless indicated otherwise in a credit line to the material. If material is not included in the article's Creative Commons license and your intended use is not permitted by statutory regulation or exceeds the permitted use, you will need to obtain permission directly from the copyright holder. To view a copy of this license, visit http://creativecommons.org/licenses/by/4.0/.

(c) The Author(s) 2020 